\title{
The Bost conjecture and proper Banach algebras
}

\author{
Walther Paravicini
}

\begin{abstract}
For proper Banach algebra coefficients, the Bost assembly map is split surjective and the right-hand side of the conjecture does not depend on the choice of the unconditional completion involved. These results hold for a large class of unconditional completions.
\end{abstract}

Mathematics Subject Classification (2010). 43A20, 22D15; 22A22; 19 K35.

Keywords. Bost conjecture, Banach algebra, proper groupoid.

In analogy to the definition of the assembly map of Baum-Connes, one can construct a homomorphism $\mu_{\mathcal{A}}^{B}$ from $\mathrm{K}_{*}^{\text {top }}(\mathcal{E}, B)$ to $\mathrm{K}_{*}(\mathcal{A}(\mathcal{E}, B))$, where $\mathcal{E}$ is a locally compact Hausdorff groupoid with Haar system, $B$ is a $\mathscr{E}-\mathrm{C}^{*}$-algebra and $\mathcal{A}(\mathscr{E})$ is an unconditional completion of $\ell_{c}(\mathscr{E})$, that is, a completion with respect to a submultiplicative norm $\|\cdot\|_{\mathcal{A}}$ such that $\|f\|_{\mathcal{A}}$ only depends on the function $\gamma \mapsto|f(\gamma)|$. This construction was discussed in [Laf06] and an obvious generalisation to $\mathcal{E}$-Banach algebras $B$ instead of $\mathscr{E}-\mathrm{C}^{*}$-algebras was given in [Par09a]. See Section 1 of the present article for a quick review.

It is well known that the Baum-Connes conjecture is true for proper $\mathrm{C}^{*}$-coefficients, and this result is a key ingredient for the so-called Dirac-dual-Dirac method, see [Kas95], which is applied to prove the conjecture for certain classes of groups and arbitrary $\mathrm{C}^{*}$-coefficients. The Baum-Connes conjecture for proper $\mathrm{C}^{*}$-coefficients is also used to show the Bost conjecture for proper $\mathrm{C}^{*}$-coefficients in [Laf02]. In the present article, we consider proper Banach algebras instead of proper $\mathrm{C}^{*}$-algebras, see Section 2 for a definition.

The main result of the present article asserts that the Bost assembly map $\mu_{\mathcal{A}}^{B}$ is split surjective if the $\mathscr{E}$-Banach algebra $B$ is proper and non-degenerate (and $\mathcal{A}(\mathscr{G})$ satisfies some mild condition), see Theorem 3.2. This is a first positive result for coefficients which are not $\mathrm{C}^{*}$-algebras. The proof does not make use of $\mathrm{C}^{*}$-algebraic methods either but rests on a generalised version of the Green-Julg theorem for Banach algebras as presented in [Par13b], which enters in Lemma 3.1:

Let $\mathcal{E}$ be proper with unit space $X$. Let $B$ be a non-degenerate $\mathcal{E}$-Banach algebra. Then we have an isomorphism (under some assumptions on $X / \mathscr{E}$ and $\mathcal{A}(\mathscr{G})$ ):

$$
\mathrm{KK}_{\mathscr{E}}^{\mathrm{ban}}\left(\boldsymbol{C}_{0}(X), B\right) \cong \mathcal{R} \mathrm{KK}^{\mathrm{ban}}\left(\boldsymbol{C}_{0}(X / \mathcal{E}) ; \boldsymbol{C}_{0}(X / \mathcal{E}), \mathcal{A}(\mathcal{E}, B)\right)
$$


A second line of reasoning in the present article investigates the dependence of the right-hand side of the Bost conjecture (with proper coefficients) on the choice of the unconditional completion, see Section 2.4 for results in this direction.

Acknowledgements. I would like to thank my Ph.D. supervisor Siegfried Echterhoff; the central results of this article are contained in my doctoral thesis [Par07]. I also thank Vincent Lafforgue, who has drawn my attention to the study of the Bost conjecture for proper Banach algebra coefficients, for his encouraging advice. And I thank the referee of this article for his helpful suggestions. This research has been supported by the Deutsche Forschungsgemeinschaft (SFB 478).

Notation. All Banach spaces and Banach algebras that appear in this article are supposed to be complex. References which explain the necessary notation and the concepts to understand Banach algebras that carry actions of groupoids are [Laf06] and [Par09a].

\section{The Bost conjecture}

In this section, let $\mathcal{E}$ be a locally compact Hausdorff groupoid equipped with a Haar system. Assume moreover that there is a locally compact classifying space E $\mathcal{E}$ for proper actions of $\mathscr{E}$, which is then unique up to homotopy. Let $\mathcal{A}(\mathscr{E})$ be an unconditional completion of $\boldsymbol{C}_{c}(\mathscr{Y})$.

If $B$ is a $\mathcal{E}$-Banach algebra, then there is an obvious definition of a $\mathcal{E}$-Banach algebra $S B:=B] 0,1\left[\right.$ with fibres $\left.B_{g}\right] 0,1\left[\right.$ for all $g \in \mathcal{E}^{(0)}$.

Definition 1.1. For every $\mathcal{G}$-Banach algebra $B$, define the topological $\mathrm{K}$-theory for $\mathcal{G}$ and $B$ as

$$
\mathrm{K}_{0}^{\mathrm{top}, \mathrm{ban}}(\mathscr{E}, B):=\lim _{\longrightarrow} \mathrm{KK}_{\mathscr{E}}^{\mathrm{ban}}\left(\mathcal{C}_{0}(X), B\right),
$$

where $X$ runs through the closed proper $\mathcal{E}$-compact subspaces of E $\mathscr{E}$. Define $\mathrm{K}_{n}^{\text {top,ban }}(\mathcal{G}, B):=\mathrm{K}_{0}^{\text {top,ban }}\left(\mathcal{E}, S^{n} B\right)$ for $n \in \mathbb{N}$.

Note that if $X$ is a locally compact Hausdorff left $\mathcal{E}$-space (with anchor map $\rho$ ), then we would like to think of $\mathcal{C}_{0}(X)$ as a $\mathscr{E}$-Banach space. We thus have to take the push-forward $\rho_{*} \mathbb{C}_{X}$ instead, which is a field over $\mathcal{E}^{(0)}$, but to make the notation more familiar, we nevertheless write $\ell_{0}(X)$. See Section 2.1 for a sketch of the push-forward construction, and for more details, consult [Par09b] or [Par07].

If $B$ is a $\mathcal{E}-C^{*}$-algebra, then there is a canonical homomorphism from the $\mathrm{C}^{*}$ algebraic version of topological K-theory to the Banach algebraic version:

$$
\mathrm{K}_{*}^{\mathrm{top}}(\mathcal{E}, B) \rightarrow \mathrm{K}_{*}^{\mathrm{top}, \mathrm{ban}}(\mathcal{E}, B) .
$$

The Bost assembly map is defined in analogy to the Baum-Connes assembly map: 
Definition 1.2. Let $B$ a $\mathscr{E}$-Banach algebra. Define the Bost assembly map as the homomorphism of abelian groups

$$
\mu_{\mathcal{A}}^{B}: \mathrm{K}_{0}^{\mathrm{top}, \mathrm{ban}}(\mathcal{E}, B) \rightarrow \mathrm{K}_{0}(\mathcal{A}(\mathcal{E}, B))
$$

which is the direct limit of the group homomorphisms $\mu_{\mathcal{A}, X}^{B}$ given by $\mathrm{KK}_{\mathscr{G}}^{\mathrm{ban}}\left(\mathcal{C}_{0}(X), B\right) \stackrel{j_{\mathcal{A}}}{\longrightarrow} \mathrm{KK}^{\mathrm{ban}}\left(\mathcal{A}\left(\mathcal{E}, \mathcal{C}_{0}(X)\right), \mathcal{A}(\mathcal{E}, B)\right) \stackrel{\Sigma(\cdot)\left(\lambda_{X, \mathcal{E}, \mathcal{A}}\right)}{\longrightarrow} \mathrm{K}_{0}(\mathcal{A}(\mathcal{E}, B))$, where $X$ runs through all closed, $\mathcal{H}$-compact, proper subspaces of $\underline{E} \mathscr{E}$.

Here $\lambda_{X, \mathscr{E}, \mathcal{A}}$ denotes a canonical element of $\mathrm{K}_{0}\left(\mathcal{A}\left(\mathscr{G}, \boldsymbol{C}_{0}(X)\right)\right)$ and $\Sigma(\cdot)$ denotes the action of $\mathrm{KK}^{\mathrm{ban}}$ on K-theory. See [Par09b] or [Par07] and to some extent [Laf06] for more details.

To define the Bost assembly map also for higher K-groups note that there is the canonical homomorphism $\iota_{B}: \mathcal{A}(\mathcal{E}, S B) \rightarrow S \mathcal{A}(\mathcal{E}, B)$ for every $\mathcal{E}$-Banach algebra $B$. For $\mathrm{K}_{1}^{\text {top,ban }}(\mathcal{G}, B)$ we can define $\mu_{\mathcal{A}}^{B}$ as the composition

$$
\begin{aligned}
\mathrm{K}_{1}^{\mathrm{top}, \mathrm{ban}}(\mathcal{E}, B)=\mathrm{K}_{0}^{\mathrm{top}, \mathrm{ban}}(\mathcal{E}, S B) & \stackrel{\mu_{\mathcal{A}}^{B}}{\longrightarrow} \mathrm{K}_{0}(\mathcal{A}(\mathcal{E}, S B)) \\
& \stackrel{\iota_{B, *}}{\longrightarrow} \mathrm{K}_{0}(S \mathcal{A}(\mathcal{E}, B))=\mathrm{K}_{1}(\mathcal{A}(\mathcal{E}, B)) .
\end{aligned}
$$

Proceed inductively to define the assembly map for all $n \in \mathbb{N}_{0}$. Note that $\iota_{B}$ is an isomorphism in K-theory by the results of [Par13a].

The Banach algebraic version of the Bost conjecture for $\mathcal{E}$ and $\mathcal{A}(\mathcal{E})$ with coefficients in a $\mathcal{G}$-Banach algebra $B$ asserts that $\mu_{\mathcal{A}}^{B}$ is an isomorphism for all $n \in \mathbb{N}_{0}$. If $B$ is a $\mathcal{E}-C^{*}$-algebra, then the assembly map introduced in [Laf06] factors through the Banach algebraic topological K-theory, i.e., it is given by the composition

$$
\mathrm{K}_{*}^{\mathrm{top}}(\mathscr{G}, B) \rightarrow \mathrm{K}_{*}^{\mathrm{top}, \text { ban }}(\mathscr{E}, B) \stackrel{\mu_{\mathscr{A}}^{B}}{\longrightarrow} \mathrm{K}_{*}(\mathcal{A}(\mathcal{E}, B)) .
$$

The $C^{*}$-algebraic version of the Bost conjecture for $\mathcal{E}$ and $\mathcal{A}(\mathcal{E})$ with coefficients in a $\mathcal{E}$-C $\mathrm{C}^{*}$-algebra $B$ asserts that the composed assembly map is an isomorphism. The $C^{*}$-algebraic version of the Bost conjecture for groups is said to be an instance of an "isomorphism conjecture" as elaborated in [BEL07].

\section{Proper Banach algebras}

2.1. Proper Banach algebras: the group case. Let $G$ be a locally compact Hausdorff group. Then a $G$-Banach algebra $B$ is called proper if there is a proper locally compact Hausdorff $G$-space $Z$ and a module action of $\ell_{0}(Z)$ on $B$ such that $B$ is a locally $\digamma_{0}(Z)$-convex $G-\bigodot_{0}(Z)$-Banach algebra, i.e., the action of $\ell_{0}(Z)$ satisfies the following conditions 
(1) $\|\chi b\| \leq\|\chi\|_{\infty}\|b\|$ for all $\chi \in \bigodot_{0}(Z), b \in B$, i.e., $B$ is a Banach $\ell_{0}(Z)$-module;

(2) the linear span of $\mathscr{C}_{0}(Z) \cdot B$ is dense in $B$, i.e., $B$ is non-degenerate as a Banach $e_{0}(Z)$-module;

(3) $\chi\left(b b^{\prime}\right)=(\chi b) b^{\prime}=b\left(\chi b^{\prime}\right)$ for all $\chi \in \bigodot_{0}(Z), b, b^{\prime} \in B$, i.e., $B$ is a $\ell_{0}(Z)$ Banach algebra;

(4) $B$ is locally $\digamma_{0}(Z)$-convex, i.e., for all $\chi_{1}, \chi_{2} \in \ell_{0}(Z), \chi_{1}, \chi_{2} \geq 0, \chi_{1}+\chi_{2} \leq 1$ and all $b_{1}, b_{2} \in B$ we have

$$
\left\|\chi_{1} b_{1}+\chi_{2} b_{2}\right\| \leq \max \left\{\left\|b_{1}\right\|,\left\|b_{2}\right\|\right\} .
$$

It is conceivable that the last condition, local $\varphi_{0}(Z)$-convexity, can be dropped, but this condition ensures that we can regard $B$ as an upper semi-continuous field of Banach algebras over $Z$, which brings this notion of a proper Banach algebra in line with the notion we define below for the groupoid case. See [Gie82] and also Appendix A. 2 of [Par07] for more information on this concept of local convexity. Because many proper $G$-Banach algebras that appear naturally are locally convex and because there is quite some machinery available to treat the general groupoid case, it seems to be advisable to postpone the non-locally $\digamma_{0}(Z)$-convex case to a later date; in any case, if one is given a possibly non-locally convex $G-\digamma_{0}(Z)$-Banach algebra $B$, one can always form and study the closely related Gelfand transform $S(B)$ which is locally $e_{0}(Z)$-convex, compare Section 1.3 of [Par13b].

2.2. Proper Banach algebras: the groupoid case. Let $\mathcal{G}$ be a locally compact Hausdorff groupoid with unit space $X$. Consider the following "mock definition" of a proper $\mathcal{E}$-Banach algebra:

"A $\mathcal{E}$-Banach algebra $B$ is called proper if there is a proper locally compact

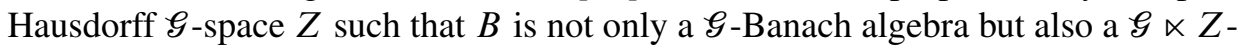
Banach algebra."

There is a little technical problem with this definition: Recall that a $\mathcal{E}$-Banach algebra is, in particular, an upper semi-continuous field of Banach algebra over $X$. So if $Z$ is a $\mathcal{E}$-space (with anchor map $\rho$ ), then the unit space of $\mathcal{Z} \ltimes Z$ is $Z$ and not $X$. As a consequence, a $\mathcal{Z} \ltimes Z$-Banach algebra is a different object than a $\mathcal{E}$-Banach algebra: to every $x \in X$, a $\mathcal{E}$-Banach algebra will have only one fibre over $x$, whereas a $\mathscr{Z} \ltimes$-Banach algebra will have a fibre for every $z \in Z$ with $\rho(z)=x$.

To be able to compare $\mathcal{E} \ltimes Z$-Banach algebras to $\mathcal{E}$-Banach algebras, it is hence necessary to introduce a process which collects all the fibres over elements $z \in$ $\rho^{-1}(\{x\}) \subseteq Z$ into a single fibre over $x$. This is done by a simple device that we call the push-forward construction. It turns a $\mathcal{E} \times Z$-Banach algebra $\hat{B}$ into a $\mathcal{E}$-Banach algebra $\rho_{*}(\widehat{B})$ and will be sketched in the next paragraph. It allows us to give the following definition.

Definition 2.1 (Proper Banach algebra). A $\mathcal{E}$-Banach algebra $B$ is called proper if there is a proper locally compact Hausdorff $\mathcal{E}$-space $Z$ (with anchor map $\rho$ ) and a 
$\mathcal{E} \ltimes Z$-Banach algebra $\widehat{B}$ such that the $\mathcal{E}$-Banach algebra $\rho_{*} \widehat{B}$ is isomorphic to $B$.

As for proper $\mathrm{C}^{*}$-algebras one can prove that we can assume without loss of generality that the space $Z$ is equal to $\underline{E} \mathcal{E}$. Note that if $\mathcal{G}$ itself is proper, then every $\mathcal{G}$-Banach algebra is proper.

2.3. The push-forward construction. We give a brief summary of the push-forward construction introduced in [Par07], Section 8.3; see also [Par09b], Section 1 and compare [CEOO03]. Let $Y$ be a locally compact Hausdorff left $\mathscr{\ell}$-space with anchor map $\rho$ and let $B$ be a $\mathscr{Y} \ltimes Y$-Banach algebra (we use the letter $Y$ instead of $Z$ to emphasise the fact that we do not assume that $Y$ is proper). We now turn $B$ into a $\mathcal{E}$-Banach algebra: For all $x \in X$, define $^{1}$

$$
\left(\rho_{*} B\right)_{x}:=\Gamma_{0}\left(Y_{x},\left.B\right|_{Y_{x}}\right)
$$

On this family $\rho_{*} B=\left(\left(\rho_{*} B\right)_{x}\right)_{x \in X}$ of Banach algebras over $X$, one can define a structure of a u.s.c. field of Banach spaces over $X$ such that $\left\{\rho_{*}(\xi): \xi \in \Gamma_{0}(Y, B)\right\}=$ $\Gamma_{0}\left(X, \rho_{*} B\right)$, where $\rho_{*}(\xi):\left.x \mapsto \xi\right|_{Y_{x}}$ for all $\xi \in \Gamma_{0}(Y, B)$. Moreover, there is a canonical $\mathscr{E}$-action on $\rho_{*} B$ making it a $\mathscr{E}$-Banach algebra. If $B$ is non-degenerate, then so is $\rho_{*} B$.

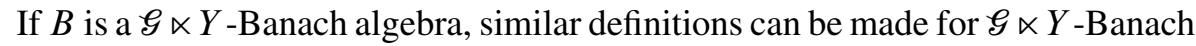
$B$-pairs to obtain $\mathscr{E}$-Banach $\rho_{*} B$-pairs. We call this construction the push-forward construction or forgetful map; the idea is that $\rho_{*}$ forgets the fine fibration over $Y$ and only remembers the coarser fibration over $X$.

The forgetful map lifts to $\mathrm{KK}^{\mathrm{ban}}$-cycles, i.e., if $A$ and $B$ be $\mathcal{E} \ltimes Y$-Banach algebras, then $\rho_{*}$ gives a homomorphism

$$
\rho_{*}: \mathrm{KK}_{\mathscr{E} \times Y}^{\mathrm{ban}}(A, B) \rightarrow \mathrm{KK}_{\mathscr{G}}^{\mathrm{ban}}\left(\rho_{*} A, \rho_{*} B\right) .
$$

This construction induces as well a homomorphism from $\mathrm{K}^{\text {top,ban }}(\mathcal{G} \ltimes Y, B)$ to $\mathrm{K}^{\text {top,ban }}\left(\mathcal{G}, \rho_{*} B\right)$.

The forgetful map is also compatible with the descent. To be more precise, let $\mathcal{A}(\mathscr{E})$ be an unconditional completion of $\mathcal{C}_{c}(\mathscr{G})$. For all $\xi \in \mathcal{C}_{c}(\mathscr{G} \ltimes Y)$, define

$$
\|\xi\|_{\mathcal{A}_{Y}}:=\left\|\gamma \mapsto \sup _{y \in Y_{r_{\mathcal{G}}(\gamma)}}|\xi(\gamma, y)|\right\|_{\mathcal{A}}
$$

This is an unconditional norm on $\mathcal{C}_{c}(\mathscr{E} \ltimes Y)$. We have $\mathcal{A}_{Y}(\mathcal{G} \ltimes Y, B) \cong \mathcal{A}\left(\mathcal{E}, \rho_{*} B\right)$ for all $\mathcal{Y} \ltimes Y$-Banach algebras $B$. This isomorphism gives us the isomorphism on the

\footnotetext{
${ }^{1}$ This definition makes sense if $x \in \rho(Y)$, and can and should be interpreted as $\rho_{*}(B)_{x}=0$ if $x \notin \rho(Y)$.
} 
right-hand side of the following commutative diagram, see [Par09b], Proposition 1.9.

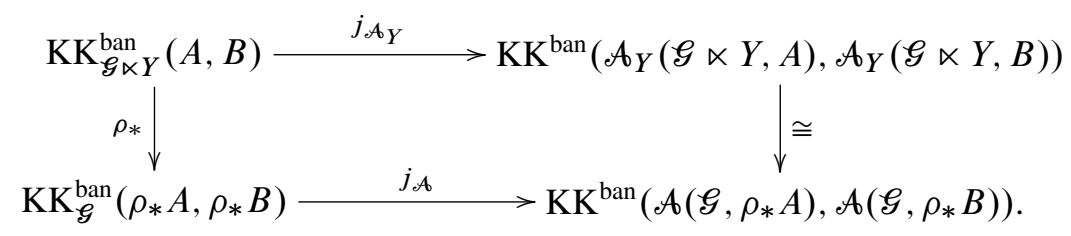

In [Par09b], it is shown that the following diagram is commutative.

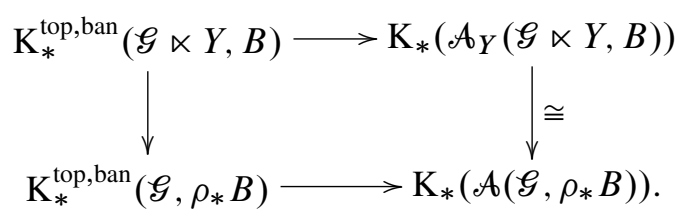

2.4. Properness and the invariance of $K$-theory. In this Section, let $\mathcal{E}$ be a locally compact Hausdorff groupoid with Haar system and unit space $X$ and let $\mathcal{A}(\mathscr{E})$ be an unconditional completion of $\mathcal{C}_{c}(\mathscr{\xi})$.

If we believe in a version of the Bost conjecture for $\mathcal{E}$ and proper Banach algebras as coefficients, we have to trust in the following conjecture about the right-hand side of the Bost assembly map.

Conjecture 2.2. Let $B$ be a proper $\mathscr{G}$-Banach algebra. Then the group $\mathrm{K}_{*}(\mathcal{A}(\mathscr{E}, B))$ is independent of the choice of the unconditional completion $\mathcal{A}(\mathscr{G})$ of $\mathcal{C}_{c}(\mathscr{E})$.

By "independent" we mean that there is a canonical isomorphism $\mathrm{K}_{*}(\mathcal{A}(\mathscr{E}, B)) \cong$ $\mathrm{K}_{*}\left(\mathcal{A}^{\prime}(\mathscr{E}, B)\right)$ whenever $\mathcal{A}^{\prime}(\mathscr{H})$ is another choice of an unconditional completion.

Note that Lafforgue has shown a result along these lines in [Laf06], namely Lemme 1.5.7 which claims that the above conjecture is true for $\mathrm{C}^{*}$-algebra coefficients. As the proof there is rather sketchy, it seems advisable to give some more explicit arguments, which are, of course, heavily influenced by Lafforgue's ideas.

In [Par13b], Appendix A, we considered a similar, seemingly weaker conjecture, namely:

Conjecture 2.3. Let $\mathcal{G}$ be proper and let $B$ be a $\mathcal{G}$-Banach algebra. Then the group $\mathrm{K}_{*}(\mathcal{A}(\mathscr{G}, B))$ is independent of the choice of the unconditional completion $\mathcal{A}(\mathscr{G})$ of $\ell_{c}(\mathscr{G})$.

In fact the two conjectures are equivalent:

If $\mathcal{E}$ is proper, then every $\mathcal{E}$-Banach algebra is proper, so Conjecture 2.3 follows from Conjecture 2.2.

On the other hand, if $B$ is a proper $\mathscr{E}$-Banach algebra, then we can find a proper locally compact Hausdorff $\mathcal{E}$-space $Z$ with anchor map $\rho$ and a $\mathcal{E} \ltimes Z$-Banach 
algebra $\widehat{B}$ such that the $\mathcal{E}$-Banach algebra $\rho_{*} \widehat{B}$ is isomorphic to $B$. If $\mathcal{A}(\mathcal{E})$ is an unconditional completion of $\mathcal{C}_{c}(\mathscr{G})$, then $\mathscr{A}_{Z}(\mathscr{G} \ltimes Z)$ is an unconditional completion of $\mathcal{C}_{c}(\mathscr{E} \ltimes Z)$, and $\mathcal{A}(\mathcal{E}, B) \cong \mathcal{A}_{Z}(\mathscr{E} \ltimes Z, \widehat{B})$. So if Conjecture 2.3 holds, then the K-theory of $\mathcal{A}_{Z}(\mathscr{E} \ltimes Z, \widehat{B})$ does not depend on the choice of the unconditional completion $\mathcal{A}_{Z}$, so the K-theory of $\mathcal{A}(\mathcal{E}, B)$ does not depend on the choice of the unconditional completion $\mathcal{A}$.

Unfortunately, we are not able to show any of these conjectures in full generality, at least for now, but in [Par13b] we obtained the following far-reaching (but still partial) result.

Proposition 2.4 ([Par13b], Proposition A.2). If $\mathcal{E}$ is proper, if $\mathcal{A}(\mathcal{E})$ is either a regular or a strictly positive unconditional completion of $\mathcal{C}_{c}(\mathscr{E})$ and if $B$ is a $\mathscr{E}$ Banach algebra, then $\mathrm{K}_{*}(\mathcal{A}(\mathscr{E}, B))$ is independent of the choice of the (regular or strictly positive) unconditional completion $\mathcal{A}(\mathscr{E})$.

The notion of regularity was defined in [Par13b]. Roughly speaking, it means that $\mathcal{A}(\mathscr{E})$ acts on a certain nice completion of $\mathcal{C}_{c}(\mathscr{E})$; for example, $\mathrm{L}^{1}(\mathscr{G})$ acts on $\mathrm{L}^{2}(\mathscr{G})$ and is therefore regular. Also every unconditional norm which dominates the reduced $\mathrm{C}^{*}$-norm on $\ell_{c}(\mathcal{E})$ is regular, and every unconditional norm that dominates another unconditional norm is regular. However, there are cases of non-regular unconditional norms even when $G$ is a group, see Example A.5 of [Par13b].

On the other hand, strict positivity is a rather technical condition which we define as follows.

Definition 2.5 ([Par13b], Definition A.3). Let $\mathcal{A}(\mathcal{E})$ be an unconditional completion of $\mathcal{C}_{c}(\mathscr{E})$ for a norm $\|\cdot\|_{\mathcal{A}}$. Then the norm $\|\cdot\|_{\mathcal{A}}$ and the completion $\mathcal{A}(\mathscr{E})$ are called strictly positive if for all compact $K \subseteq X$ there is a function $\chi \in \mathcal{C}_{c}(\mathscr{E})_{\geq 0}$ such that $\inf _{x \in K}\|\chi\|_{\mathcal{A}_{x}}>0$.

Note that if $\mathscr{E}=G$ is a group, then every unconditional norm on $\mathcal{C}_{c}(G)$ is strictly positive. Secondly, note that $\mathrm{L}^{1}(\mathcal{E})$ is both, strictly positive and regular. But there are also regular unconditional completions which are not strictly positive, and vice versa, see [Par13b], Example A.5.

We can generalise Proposition 2.4 to the following result on proper Banach algebras:

Proposition 2.6. If $\mathcal{A}(\mathcal{G})$ is either a regular or a strictly positive unconditional completion of $\mathcal{C}_{c}(\mathcal{E})$ and if $B$ is a proper $\mathcal{E}$-Banach algebra, then $\mathrm{K}_{*}(\mathcal{A}(\mathcal{H}, B))$ is independent of the choice of the (regular or strictly positive) unconditional completion $\mathcal{A}(\mathcal{E})$.

It is clear that this proposition implies Proposition 2.4. But also the reverse implication is true, which can be deduced from the following observation. 
Lemma 2.7. Let $Y$ be a locally compact Hausdorff $\mathscr{E}$-space. If $\mathcal{A}(\mathscr{E})$ is regular, then $\mathcal{A}_{Y}(\mathscr{G} \ltimes Y)$ is regular. And if $\mathcal{A}(\mathscr{G})$ is strictly positive, then $\mathcal{A}_{Y}(\mathscr{E} \ltimes Y)$ is strictly positive.

Proof. The part concerning regularity was settled in Proposition 8.3.26 of [Par07]; we do not reproduce the straightforward but somewhat technical proof here.

We sketch the proof for the part about strict positivity. So assume that $\mathcal{A}(\mathscr{E})$ is strictly positive. Let $L \subseteq Y$ be compact. Define $K:=\rho(L)$. This is a compact subset of $\mathscr{E}^{(0)}$, so there is a function $\chi_{\mathcal{E}} \in \mathcal{C}_{c}(\mathscr{E})_{\geq 0}$ such that inf $\operatorname{coK}_{x \in K}\left\|\chi_{\mathcal{E}}\right\|_{\mathcal{A}_{x}}>0$. Find a function $\chi_{Y} \in \mathscr{\ell}_{c}(Y)$ such that $\chi_{Y}(y)=1$ on some open neighbourhood of $L$ and such that $0 \leq \chi(y) \leq 1$ for all $y \in Y$. Define

$$
\chi_{\mathscr{E} \ltimes Y}(\gamma, y):=\chi_{\mathscr{E}}(\gamma) \chi_{Y}(y), \quad(\gamma, y) \in \mathcal{E} \ltimes Y .
$$

Then $\chi_{\varepsilon_{\ltimes}}$ is continuous with compact support and satisfies, for all $y \in L$,

$$
\|\chi \mathscr{E} \ltimes Y\|_{\left(\mathcal{A}_{Y}\right)_{y}}=\|\chi \mathscr{E}\|_{\mathcal{A}_{\rho(y)}} .
$$

Hence

$$
\inf _{y \in L}\|\chi \mathscr{E} \ltimes Y\|_{\left(\mathcal{A}_{Y}\right)_{y}} \geq \inf _{x \in K}\|\chi \mathscr{g}\|_{\mathcal{A}_{x}}>0 .
$$

Finally, if $\mathscr{E}=G$ is a group, then every unconditional completion of $\ell_{c}(G)$ is strictly positive, so we obtain the following corollary to Proposition 2.6 (for which Lafforgue has indicated me a more direct proof which served as a basis for some of the arguments developed in Appendix A of [Par13b]):

Corollary 2.8. If $\mathscr{G}=G$ is a locally compact group and if $B$ is a proper $G$-Banach algebra, then $\mathrm{K}_{*}(\mathcal{A}(G, B))$ is independent of the choice of the unconditional completion $\mathcal{A}(G)$.

\section{The Bost conjecture and proper Banach algebras}

In this section, let $\mathcal{E}$ be a locally compact Hausdorff groupoid equipped with a Haar system. Assume moreover that there is a locally compact classifying space $\underline{E} \mathcal{E}$ for proper actions of $\mathscr{G}$, which is then unique up to homotopy. Let $\mathcal{A}(\mathscr{G})$ be an unconditional completion of $\mathcal{C}_{c}(\mathscr{E})$.

3.1. The Bost conjecture and proper groupoids. We first consider the case that $\mathscr{E}$ itself is proper. In this case, the proper $\mathscr{G}$-space $X=\mathscr{E}^{(0)}$ is a model for $\underline{E} \mathscr{E}$. If, in addition, $X / \mathscr{E}$ is compact, then the canonical homomorphism

$$
\mathrm{KK}_{\mathscr{g}}^{\mathrm{ban}}\left(\mathcal{C}_{0}(X), B\right) \rightarrow \mathrm{K}_{0}^{\mathrm{top}, \mathrm{ban}}(\mathscr{E}, B)
$$


is an isomorphism; moreover, the following diagram commutes:

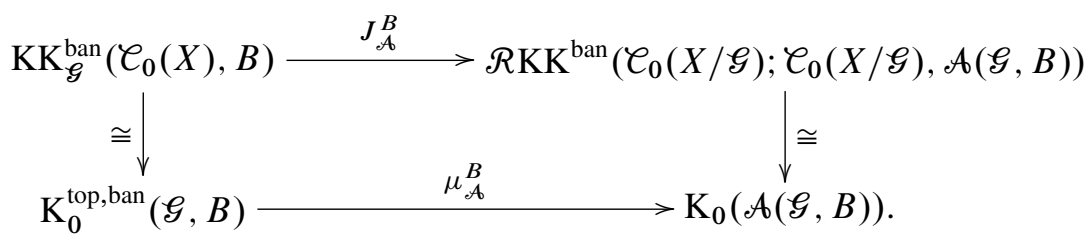

The isomorphism on the right-hand side is the given by the embedding $\mathbb{C} \mapsto \boldsymbol{C}_{0}(X / \mathcal{G})$ as constant functions, see Corollary 1.10 of [Par13b]. Actually, diagram (2) commutes already on the level of ( $\mathscr{E}$-equivariant) $\mathrm{KK}^{\mathrm{ban}}$-cycles up to isomorphism.

Applying the genereralised Green-Julg Theorem for proper groupoids and Banach algebra coefficients, we can infer that the top arrow of diagram (2) is an isomorphism, see Corollary 2.5 of [Par13b].

Lemma 3.1. Let $\mathcal{E}$ be proper, let $X / \mathcal{G}$ be compact, let $\mathcal{A}(\mathcal{E})$ be regular or strictly positive and let $B$ be non-degenerate. Then the Bost map $\mu_{\mathcal{A}}^{B}$ is an isomorphism.

Note that diagram (2) gives the result directly only for $*=0$, but we can apply it to $S^{n} B$ instead of $B$ and use the invariance results for K-theory of [Par13a] to obtain it for arbitrary degrees.

3.2. The Bost conjecture and proper Banach algebras. Let $B$ be a non-degenerate proper $\mathcal{E}$-Banach algebra and let $\mathcal{A}(\mathcal{E})$ be a regular or strictly positive unconditional completion of $\boldsymbol{C}_{c}(\boldsymbol{G})$.

Theorem 3.2. The homomorphism

$$
\mu_{\mathcal{A}}^{B}: \mathrm{K}_{*}^{\text {top,ban }}(\mathscr{E}, B) \rightarrow \mathrm{K}_{*}(\mathcal{A}(\mathcal{E}, B))
$$

is split surjective. The split is natural in $B$.

This applies in particular to the regular unconditional completion $\mathrm{L}^{1}(\mathcal{E})$ and its symmetrised version $\mathrm{L}^{1}(\mathcal{E}) \cap \mathrm{L}^{1}(\mathcal{E})^{*}$.

Before we prove Theorem 3.2, we consider yet another special case:

Lemma 3.3. Let $B$ be a non-degenerate proper $\mathcal{G}$-Banach algebra such that there exists a proper $\mathcal{E}$-compact $\mathcal{E}$-space $Z$ with anchor map $\rho$ and $a \mathscr{E} \ltimes Z$-Banach algebra $\widehat{B}$ such that $\rho_{*} \widehat{B} \cong B$. Then $\mu_{\mathcal{A}}^{B}$ is split surjective, the split being natural in $B$.

Proof. Let $Z, \rho$ and $\widehat{B}$ be as in the statement of the lemma. By Lemma 2.7, $\mathcal{A}_{Z}\left(\mathscr{E}_{\ltimes} Z\right)$ is a regular or strictly positive unconditional completion of $\mathcal{C}_{c}(\mathscr{E} \ltimes Z)$ because $\mathcal{A}(\mathscr{G})$ is regular or strictly positive. So by Lemma 3.1, the homomorphism

$$
\mu_{\mathcal{A}_{Z}}^{\widehat{B}}: \mathrm{K}_{*}^{\text {top,ban }}(\mathcal{E} \ltimes Z, \widehat{B}) \rightarrow \mathrm{K}_{*}\left(\mathcal{A}_{Z}(\mathscr{G} \ltimes Z, \widehat{B})\right)
$$


is an isomorphism. The diagram

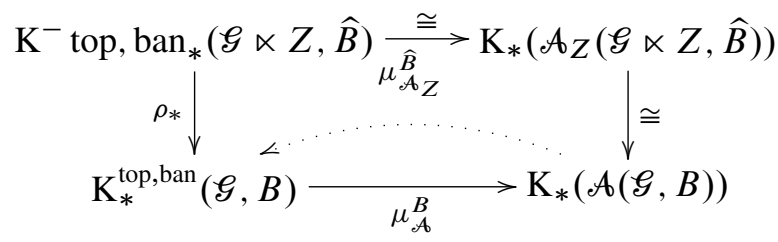

commutes, see diagram (1) above. Because the top arrow and the right-hand arrow are natural isomorphisms and the left-hand arrow is natural, too, the bottom arrow has a natural split.

Proof of Theorem 3.2. Let $\widehat{B}$ be a $\mathscr{E} \ltimes \mathrm{E} \mathcal{E}$-Banach algebra and let $\rho: \mathrm{E} \mathscr{E} \rightarrow X=$ $\mathscr{E}^{(0)}$ be the anchor map of the proper action of $\mathcal{G}$ on $\underline{E}$; assume that $\rho_{*} \widehat{B} \cong B$ as $\mathcal{E}$-Banach algebras. Then $\widehat{B}$ is non-degenerate. For every open $\mathcal{E}$-invariant subspace $U$ of $\underline{E} \mathscr{\mathscr { S }}$, define $\widehat{B}_{U}$ to be the $\mathscr{E} \ltimes \underline{\mathrm{E}} \mathcal{\mathscr { S }}$-Banach algebra with the following fibres: If

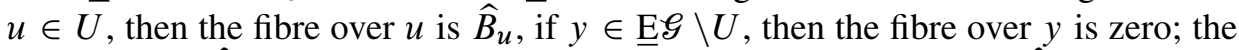
space $\Gamma\left(\underline{E} \mathcal{E}, \widehat{B}_{U}\right)$ is defined to be the set of all elements of $\Gamma(\underline{E} \mathcal{E}, \widehat{B})$ that vanish outside $U$. By definition, there is a $\mathscr{E} \ltimes \mathrm{E} \mathscr{E}$-equivariant "injection" $\hat{\jmath}_{U}$ from $\hat{B}_{U}$ to $\widehat{B}$. It descends to a $\mathscr{E}$-equivariant homomorphism $j_{U}:=\rho_{*} \hat{\jmath}_{U}$ from $B_{U}:=\rho_{*} \widehat{B}_{U}$ to $B=\rho_{*} \widehat{B}$. We can regard $B_{U}$ as a subalgebra of $B$.

The $\widehat{B}_{U}$, where $U$ runs through the open $\mathcal{E}$-invariant subsets of $\underline{E} \mathcal{E}$ such that

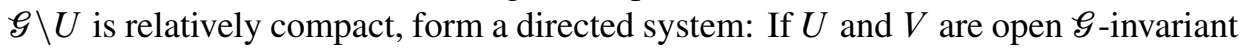
and $\mathcal{G}$-relatively compact subsets of EE with $U \subseteq V$, then there is an obvious homomorphism $\hat{\jmath}_{U, V}: \widehat{B}_{U} \rightarrow \widehat{B}_{V}$ such that $\hat{\jmath}_{U}=\overline{\hat{\jmath}}_{V} \circ \hat{\jmath}_{U, V}$. Also the $B_{U}$ form a directed system, just take the $j_{U, V}:=\rho_{*} \hat{J}_{U, V}$ as connecting maps. We can regard $B$ as the direct limit of the $B_{U}$. More importantly, the $\mathcal{A}\left(\mathscr{E}, B_{U}\right)$ form a directed system with connecting maps $\alpha_{U, V}:=\mathcal{A}\left(\mathcal{E}, j_{U, V}\right): \mathcal{A}\left(\mathcal{E}, B_{U}\right) \rightarrow \mathcal{A}\left(\mathcal{E}, B_{V}\right)$. The Banach algebra $\mathcal{A}(\mathscr{E}, B)$ is the direct limit of this system with embeddings $\alpha_{U}:=$ $\mathcal{A}\left(\mathscr{E}, j_{U}\right): \mathcal{A}\left(\mathscr{G}, B_{U}\right) \rightarrow \mathcal{A}(\mathscr{E}, B)$. Because the K-theory of Banach algebras is continuous, we get:

$$
\mathrm{K}_{*}(\mathcal{A}(\mathscr{E}, B))=\underline{\lim } \mathrm{K}_{*}\left(\mathcal{A}\left(\mathscr{E}, B_{U}\right)\right)
$$

where $U$ runs through the $\mathscr{E}$-invariant open subsets of $\underline{E}$ such that $\mathscr{Y} \backslash U$ is relatively compact.

Now let $U$ be such a set. Find a closed set $Z \subseteq \mathrm{E} \mathscr{E}$ such that $U \subseteq Z$ and $\mathscr{E} \backslash Z$ is compact. Define $\rho^{Z}:=\left.\rho\right|_{Z}$. Then $\left.\hat{B}_{U}\right|_{Z}$ is a $\mathscr{E} \ltimes Z$-Banach algebra and $\left(\rho^{Z}\right)_{*} \widehat{B}_{U} \mid Z$ is isomorphic to $B_{U}$. So $B_{U}$ satisfies the hypotheses of Lemma 3.3, so $\mu_{\mathcal{A}}^{B_{U}}: \mathrm{K}_{*}^{\text {top,ban }}\left(\mathscr{E}, B_{U}\right) \rightarrow \mathrm{K}_{*}\left(\mathcal{A}\left(\mathscr{G}, B_{U}\right)\right)$ is split surjective. Let $\sigma_{U}$ denote the natural split constructed above. It is easy to see, using the naturality of the split, that $\sigma_{V} \circ\left(\alpha_{U, V}\right)_{*}=\left(j_{U, V}\right)_{*} \circ \sigma_{U}$. Define $\tau_{U}:=\left(j_{U}\right)_{*} \circ \sigma_{U}: \mathrm{K}_{*}\left(\mathcal{A}\left(\mathscr{G}, B_{U}\right)\right) \rightarrow$ $\mathrm{K}_{*}^{\text {top,ban }}(\mathcal{E}, B)$. Then $\tau_{V}=\tau_{U} \circ\left(\alpha_{U, V}\right)_{*}$. The universal property of the direct limit 
shows that there exists a natural homomorphism $\tau: \mathrm{K}_{0}(\mathcal{A}(\mathcal{G}, B)) \rightarrow \mathrm{K}^{\text {top,ban }}(\mathcal{G}, B)$ such that $\tau \circ\left(\alpha_{U}\right)_{*}=\tau_{U}$ for all $U$.

Note that

$$
\mu_{\mathscr{A}}^{B} \circ \tau_{U}=\mu_{\mathscr{A}}^{B} \circ\left(j_{U}\right)_{*} \circ \sigma_{U}=\left(\alpha_{U}\right)_{*} \circ \mu_{\mathscr{A}}^{B_{U}} \circ \sigma_{U}=\left(\alpha_{U}\right)_{*}
$$

because $\sigma_{U}$ is a split. Passing to the limit shows that $\mu_{\mathscr{A}}^{B} \circ \tau=$ id, i.e., $\tau$ is a natural split.

3.3. The case of locally compact groups. Let $G$ be a locally compact Hausdorff group. Then we have the following corollary of Theorem 3.2 and Corollary 2.8. Note that there are no conditions on the unconditional completion $\mathcal{A}(G)$; in particular, it does not have to be regular, but it will be strictly positive, automatically.

Corollary 3.4. If $B$ is a proper $G$-Banach algebra and if $\mathcal{A}(G)$ is an unconditional completion of $\mathcal{C}_{c}(G)$, then

$$
\mu_{\mathcal{A}}^{B}: \mathrm{K}_{*}^{\text {top,ban }}(G, B) \rightarrow \mathrm{K}_{*}(\mathcal{A}(G, B))
$$

is split surjective. In particular, this is true for $\mathcal{A}(G)=\mathrm{L}^{1}(G)$. Moreover, the right-hand side is independent of the choice of the unconditional completion $\mathcal{A}(G)$.

\section{References}

[BEL07] A. Bartels, S. Echterhoff, and W. Lück, Inheritance of isomorphism conjectures under colimits. In $K$-theory and noncommutative geometry, EMS Ser. Congr. Rep., Eur. Math. Soc., Zürich 2008, 41-70. Zbl 1159.19005 MR 2513332

[BCH94] P. Baum, A. Connes, and N. Higson, Classifying space for proper actions and $K$-theory of group $C^{*}$-algebras. In $C^{*}$-algebras: 1943-1993 (San Antonio, TX, 1993), Contemp. Math. 167, Amer. Math. Soc., Providence, RI, 1994, 240-291. Zbl 0830.46061 MR 1292018

[CEOO03] J. Chabert, S. Echterhoff, and H. Oyono-Oyono, Shapiro's lemma for topological K-theory of groups. Comment. Math. Helv. 78 (2003), 203-225. Zbl 1030.19001 MR 1966758

[Gie82] G. Gierz, Bundles of topological vector spaces and their duality. Lecture Notes in Math. 955, Springer-Verlag, Berlin 1982. Zbl 0488.46060 MR 674650

[Kas95] G. G. Kasparov, $K$-theory, group $C^{*}$-algebras, and higher signatures (conspectus). In Novikov conjectures, index theorems and rigidity, Vol. 1 (Oberwolfach, 1993), London Math. Soc. Lecture Note Ser. 226, Cambridge University Press, Cambridge 1995, 101-146. Zbl 0957.58020 MR 1388299

[KS03] G. Kasparov and G. Skandalis, Groups acting properly on "bolic" spaces and the Novikov conjecture. Ann. of Math. (2) 158 (2003), 165-206. Zbl 1029.19003 MR 1998480 
[Laf02] V. Lafforgue, $K$-théorie bivariante pour les algèbres de Banach et conjecture de Baum-Connes. Invent. Math. 149 (2002), 1-95. Zbl 1084.19003 MR 1914617

[Laf06] V. Lafforgue, $K$-théorie bivariante pour les algèbres de Banach, groupoïdes et conjecture de Baum-Connes. J. Inst. Math. Jussieu 6 (2007), 415-451.Zbl 1135.19002 MR 2329760

[Par07] W. Paravicini, KK-theory for Banach algebras and proper groupoids. $\mathrm{PhD}$ thesis, Universität Münster, Münster 2007. Zbl 1131.19002 http://miami.uni-muenster.de/servlets/DocumentServlet?id=3482

[Par09a] W. Paravicini, Induction for Banach algebras, groupoids and $\mathrm{KK}^{\text {ban }}$. J. K-Theory 4 (2009), 405-468. Zbl 1189.19004 MR 2570950

[Par09b] W. Paravicini, The Bost conjecture, open subgroups and groups acting on trees. $J$. K-Theory 4 (2009), 469-490. Zbl 1189.19005 MR 2570951

[Par13a] Walther Paravicini. The spectral radius in $\ell_{0}(X)$-Banach algebras. J. Noncommut. Geom. 7 (2013), 135-147.

[Par13b] W. Paravicini, A generalised Green-Julg theorem for proper groupoids and Banach algebras. J. Noncommut. Geom. 7 (2013), 149-190.

Received December 2, 2010

W. Paravicini, Mathematisches Institut, Universität Münster, Einsteinstr. 62, 48149 Münster, Germany

E-mail: W.Paravicini@uni-muenster.de 